Unfallchirurg 2009 $\cdot 112: 542$

DOI 10.1007/s00113-009-1654-6

(c) Springer Medizin Verlag 2009

\section{P.M. Vogt · C. Krettek}

Klinik und Poliklinik für Plastische, Hand- und Wiederherstellungschirurgie, Unfallchirurgische Klinik, Medizinische Hochschule Hannover

\title{
Versorgungsstandards der Verbrennungsmedizin, Teil 2
}

Im notfallmedizinischen Patientenkollektiv sind Verbrennungen mit einem Anteil von nur ca. $1 \%$ der Einsätze relativ selten, wobei die schweren, lebensbedrohlichen Brandverletzungen noch deutlich seltener als Bagatellverletzungen sind. Für den Notarzt und die weiterbehandelnden Kollegen in erstversorgenden Häusern können diese Umstände eine eingeschränkte Routine beim Umgang mit Brandverletzungen und ihren manchmal erheblichen systemischen Auswirkungen zur Folge haben. Nicht zuletzt wird das Management Brandverletzter nicht selten durch mechanische Begleitverletzungen (thermomechanisches Kombinationstrauma) weiter erschwert. In den Leitthemenbeiträgen der vorangegangenen Ausgabe wurde gezeigt, dass durch ein klar gegliedertes problemorientiertes Behandlungskonzept in der präklinischen und klinischen Notfallversorgung sowie auf der Intensivstation die entscheidenden Grundsteine für den weiteren Verlauf gelegt werden.

Nach der Intensivbehandlung stellen Rehabilitation und Rekonstruktion die wesentliche Basis der Integration brandverletzter Patienten dar. Wenngleich aufwändige rekonstruktive Eingriffe im Spätverlauf oftmals beeindruckende Ergebnisse bringen, können sie doch nicht das Versäumte in der initialen chirurgischen Versorgung für brandverletzte Patienten kompensieren. Insofern ist der fachkundige Einsatz z. B. von Hautersatzverfahren entscheidend für den weiteren Verlauf. Zu spät operativ behandel- te und nicht adäquat durch Hautersatzmaterialien oder Transplantate rekonstruierte Verbrennungen resultieren unweigerlich in kontrakten, funktionsbehindernden Narben und an exponierten Stellen darüber hinaus zu ästhetischen Entstellungen. Somit ist klar festzustellen, dass eine qualifizierte Erstbehandlung den Bedarf an späterer Rekonstruktion minimieren kann.

Eine funktionsgerechte wiederherstellende plastische Chirurgie, nicht zuletzt auch aus ästhetischer Indikation heraus, stellt für viele Patienten eine hoffnungsvolle Perspektive ihres schweren Traumas dar. Die moderne plastische Chirurgie hat hierzu über viele Jahrzehnte eine erfolgreiche Weiterentwicklung betrieben. Auf der rein operativ-technischen Seite stehen mikrovaskuläre Transplantate und präfabrizierte Gewebetransfers auch für komplexeste Deformitäten zur Verfügung. Parallel dazu erlaubt der stetig weiterentwickelte moderne Hautersatz eine sehr differenzierte Behandlung, um so eine stadien- und anatomiegerechte Wiederherstellung zu erreichen.

In diesem Themenheft zur Behandlung Schwerbrandverletzter werden der Hautersatz ebenso wie die frühe Rekonstruktion und Spätrekonstruktion und auch die funktionell bedeutsame Wiederherstellung der verbrannten Hand ausführlich beschrieben. Den betroffenen Patienten stehen heute bundesweit Brandverletztenzentren mit plastisch-rekonstruktiver Chirurgie zur Verfügung, um jederzeit entsprechend fachgerecht versorgt zu werden. Die von der DGU initiierte und weitestgehend umgesetzte Traumanetzwerkbildung ist hier ein weiterer wichtiger Baustein, um die flächendeckende und interdisziplinäre Versorgung Brandverletzter frühestmöglich und bestqualifiziert zu gewährleisten.

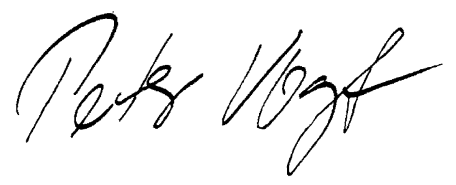

Prof. Dr. P. M. Vogt

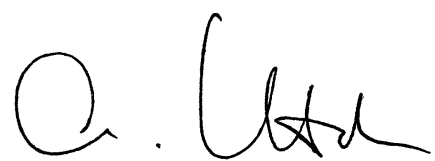

Prof. Dr. C. Krettek, FRCS, FRACS

\section{Korrespondenzadresse \\ Prof. Dr. P.M. Vogt}

Klinik und Poliklinik für Plastische, Hand- und Wiederherstellungschirurgie, Unfallchirurgische Klinik,

Medizinische Hochschule Hannover

Carl-Neuberg-Str. 1, 30625 Hannover

Vogt.Peter@MH-Hannover.de 